\section{PROTEINS}

\section{Going Native}

from our Molecular Biology Correspondent

IF one were to judge from the results of equilibrium experiments alone, one would conclude that the denaturation and refolding of at least the smaller proteins generally involves only two states, with no intermediates of appreciable weight. Ultimately this first approximation to the truth, useful as it has been, is altogether too primitive, for perhaps the most absorbing problem in protein chemistry just now is the resolution of the events that constitute the folding pathway.

A notion which has had wide currency for some time is that the rate-limiting step in the folding process is the formation of structural nuclei (the nature of which has also been the subject of much discussion), which then direct the ensuing structural condensation, according to a series of rapid reactions. Such a scheme has several attractive features, and explains in particular the strong cooperativity which is universally observed in denaturation-renaturation equilibria, as well as the conspicuously small entropy of activation for folding, which could not be easily reconciled with a rate-limiting step involving extensive conformational immobilization. Among the difficulties that arise in the interpretation of kinetics of denaturation is that of deciding whether the pathways are in all cases unique, or whether parallel processes occur, and also whether slow steps might arise from dead-end pathways which lead to incorrect conformational states, and which have to be retraced to permit a new start. Criteria for discriminating between such possibilities were not long ago formulated by Ikai and Tanford. The most profound investigation of the kinetics of the folding process has come from Baldwin's laboratory, and, with ribonuclease as a model, has for the first time penetrated to this level of complexity, and demonstrated the existence of a slow nucleation reaction.

In following the kinetics of conformational processes, it is necessary to make use of some kind of spectroscopic manifestation, which will in general contain contributions from several structural elements. Thus it is difficult to be sure that the separable steps in the kinetics of ribonuclease denaturation, followed in terms of the development of the aromatic difference spectrum, do not arise from differences between the rates of exposure of the various residues. With this in view, Tsong and Baldwin (J. Mol. Biol., 69, $149 ; 1972$ ) have examined the kinetics using a dinitrophenyl derivative of ribonuclease. The substituent is on
Lys-41, which is one of the two lysines on either side of the active site, and leaves the protein conformationally and functionally intact. On denaturation, the absorption band of the new chromophore at $360 \mathrm{~nm}$ generates its own difference spectrum, in terms of which the kinetics can be measured. In temperature-jump experiments the dinitrophenyl group produces an effect in the microsecond range, which is simply a thermal perturbation in the chromophore itself, followed by a relaxation in the millisecond range, which is essentially identical with that observed when measurements are made at a tyrosinesensitive wavelength either in the dinitrophenyl or unmodified ribonuclease. Again, the slow (rate determining) unfolding reaction, which is conveniently observed in $p \mathrm{H}$-jump experiments in the acid-unfolding range, proceeds at the same rate irrespective of the wavelength. These results reinforce confidence in the procedure, and affirm that disparate parts of the molecule share the same folding pathway. Moreover the rapid phase of the reaction is observed only in experiments well within the temperature range of the folding transition, which confirms that they are identifiable with the folding process.

A deeper examination of the ribonuclease equilibrium is now possible. Tsong, Baldwin and Elson (Proc. US Nat. Acad. Sci., 69, 1809; 1972) have been able to show that the kinetics are consistent with a sequential process of nucleation and propagation, rather than a superimposition of incorrect, abortive folding reactions. Stopped-flow measurements of $p \mathrm{H}$-dependent denaturation and refolding experiments reveal a fast process in the folding reaction only in the lower part of the $p \mathrm{H}$-transition range, whereas it persists over the whole range in the unfolding process under the same conditions. It is also important, and was demonstrated by Summers and McPhie (Biochem. Biophys. Res. Commun., 47, 831 ; 1972), and now reaffirmed by Tsong et al. that above the transition zone the unfolding reaction can essentially be expressed by the fast process alone. This all follows expectation for a sequential model. No rapid steps preceding nucleation can be detected in the range where this step is dominant, and only a low extent of folding is achieved. The rate constant for the slow phase is the same in unfolding and refolding experiments. Experiment and theory for the sequential model can be matched for a series of kinetic criteria. Tsong et al. nevertheless urge caution. Although the results can be fitted well enough (although not necessarily uniquely) in terms of the usual set of independent thermodynamic variables, they point out that the implicit assumption of a set of rapid folding or unfolding steps with identical rate constants is unrealistic; that one cannot at this stage determine whether several pathways to the same end product may exist; that the further implicit assumption of a constant heat of unfolding is barely applicable in a complex situation involving hydrophobic and other forms of interaction with specific heat components, and finally that under some conditions, below the transition zone, an unexplained intermediate phase appears in the kinetics. As the rapid phase becomes dominant in the upper part of the range, one may entertain the hope that

\title{
Replication of Adenovirus 5 DNA
}

Although most microbial and viral systems in which DNA replication has been investigated in any detail have been found to contain circular replicative intermediates, it would be quite wrong to conclude that the generation of circular molecules is a universal step in DNA replication. For it is known that all the replicative intermediates of, for example, T7 coliphage DNA are linear and according to Sussenbach, van der Vliet, Ellens and Jansz, whose latest experiments are reported in next Wednesday's Nature New Biology (September 13), the same is true of replicating adenovirus 5 DNA molecules.

This Dutch group has devised a way of isolating the nuclei of $\mathrm{KB}$ cells infected by adenovirus 5 which continue to support the replication of the viral DNA in vitro. Various adenovirus 5 DNAs with sedimentation coefficients of $31 \mathrm{~S}-60 \mathrm{~S}$ and buoyant densities of 1.716 (mature viral DNA) to 1.724 $\mathrm{g} \mathrm{cm}^{-3}$ can be isolated. The replicative intermediates with the high buoyant densities were found to contain single stranded DNA, which accounts for their buoyant density, and when they were separated from the non-replicating DNA and examined under an electron microscope they appeared to be linear molecules which fall into two chief classes.

One class contains $Y$ shaped molecules, one arm of the $Y$ being single stranded and the other arm and stem double stranded. The second class includes partially double stranded and partially single stranded, but unbranched, molecules. These and the various other forms can be explained by postulating that adenovirus 5 DNA replication starts at the end of one strand and is accompanied by the displacement of a strand; the displaced strand can then act as a template, either before or after it is completely displaced. 